\title{
Two-Tube Multiplex PCR for Genotyping Tuberculous and Nontuberculous Mycobacterial Species in Pathology Specimens
}

\author{
Michael Dictor ${ }^{1,2}$, Janina Warenholt ${ }^{1}$, Marta Lukasiewicz ${ }^{1}$ \\ ${ }^{1}$ Department of Pathology, Skåne University Hospital, Lund, Sweden \\ ${ }^{2}$ Present Address: Telemedicine Clinic, Barcelona, Spain \\ Email: michael.dictor@med.lu.se
}

How to cite this paper: Dictor, M., Warenholt, J. and Lukasiewicz, M. (2018) Two-Tube Multiplex PCR for Genotyping Tuberculous and Nontuberculous Mycobacterial Species in Pathology Specimens. Advances in Microbiology, 8, 31-41. https://doi.org/10.4236/aim.2018.81003

Received: December 18, 2017

Accepted: January 28, 2018

Published: January 31, 2018

Copyright $\odot 2018$ by authors and Scientific Research Publishing Inc. This work is licensed under the Creative Commons Attribution International License (CC BY 4.0).

http://creativecommons.org/licenses/by/4.0/

\begin{abstract}
Background: The incidence of mycobacterial infection, in particular $M$. tuberculosis complex (MTC), is increasing in some Western countries, while nontuberculous mycobacteria (NTM) may be recognized more frequently in clinical specimens worldwide. The clinical scenario and available histopathology alone are often insufficient to separate these two categories of mycobacterial disease, whose behavior and treatment differ. In particular, NTM may be clinically unsuspected in pathological specimens and the opportunity for culturing missed. Methods: We developed two multiplex PCR assays, which distinguish MTC from NTM by detecting the IS6110 insert in the first tube and discriminating up to 14 NTM reference strains in the second by targeting the 16S-23S rRNA internal transcribed spacer. Test material included 594 routine clinical specimens with diverse pathology; many were granulomas unrelated to mycobacterial infection. About $75 \%$ were formalin-fixed paraffin blocks, the remainder mainly cytologic imprints or aspirates on FTA cards submitted on suspicion of mycobacterial infection either to avoid frozen sectioning (with the attendant risk of aerosolisation) or at the time of fine needle aspiration. Results: The paraffinized material yielded 53 MTC positives and the cytological 21 positives. A subset consisting of 337 specimens was also analyzed for NTM and yielded 51 positives. The frequency of simultaneous NTM infection in tuberculous patients was about $17 \%$. Mycobacterium avium complex represented the dominant NTM species overall, showed a predilection for lung and lymph node, and together with $M$. haemophilum were the second most frequent NTM just behind $M$. ulcerans/ $M$. marinum in skin and soft tissue, the category displaying the largest NTM diversity. Conclusions: Cytological and deparaffinized tissue analyzed in a new two-tube multiplex PCR allows for specific discrimination of causative agents in mycobacterial infection.
\end{abstract}


MTC is readily distinguished from NTM for appropriate therapy, and NTM presumptively diagnosed at the species level allows appropriate choices of antimicrobials.

\section{Keywords}

Mycobacteria, Multiplex PCR, Speciation, Paraffinized Tissue, FTA Card

\section{Introduction}

Mycobacterial infection in western countries is increasingly common as a result of greater immigration from areas with a high incidence of tuberculosis, modern medicine's growing reliance on therapies with immunosuppressive effects and the use of aggressive treatments in aging populations. In the clinic, mycobacterial infection may be often overlooked and first suggested in a biopsy report at which time the only material available for culture may have been removed, fixed in formalin and embedded in paraffin. Acid-fast staining (AFS) in histological sections offers notoriously low sensitivity [1] and does not confirm the species. Clinical findings, including skin tests with PPD (purified protein derivative), in localized or disseminated infection do not always allow for the critical distinction of nontuberculous mycobacterial (NTM) disease from tuberculosis (MTC, M. tuberculosis complex). Moreover, knowledge of the specific organism in NTM infection is useful for the clinician in the choice of increasingly differentiated anti-mycobacterial therapies [2] [3].

To expedite the diagnosis of species commonly reported in human infection worldwide, in Europe or associated with immunodeficiency, as stated by the American Thoracic Society and Infectious Disease Societies of America [2], we developed a two-tube multiplex PCR (MPCR) with product detection by four-color capillary gel electrophoresis. Fragment sizes ranged from 90 to 158 bp, well suited for amplification of partly formalin-degraded DNA.

\section{Methods}

\subsection{Reference Strains}

DNA or biomass was obtained from Deutsche Sammlung von Mikroorganismen und Zellkulturen $\mathrm{GmbH}$, Braunschweig, Germany, for the following reference strains (with catalog numbers): M. bovis (43990), M. avium (43216), $M$. malmoense (44163), M. kansasii (43224), M. fortuitum (43075), M. chelonae (43217), M. haemophilum (44634), M. mucogenicum (44124). M. marinum (44344), M. gordonae (44160), M. scrofulaceum (43992), M. terrae (43227) and M. xenopi (43995).

\subsection{Clinical Specimens}

594 specimens were liberally submitted between 2010 and 2015 for MPCR by re- 
gional pathologists with variable experience in the histopathology of mycobacterial infection. Many specimens entailed simply foreign body or non-mycobacterial granulomas, while occasional cases were clinically suspected but lacked histological signs of granulomatous inflammation; all were analyzed for MTC. Of these, 337 specimens were further analyzed for NTM. Formalin-fixed paraffin-embedded (FFPE) tissue blocks accounted for $75 \%$ of submissions and the remainder were largely FTA cards imprinted with either cut unfixed tissue surface, material pelleted from cytologic fluids at the time of accession or fine needle aspirates ejected onto FTA cards, as previously described [4]. Several specimens were fluids submitted in a methanol-containing preservative for liquid cytology. The FTA card ruptures the mycobacterial cell wall on contact and binds released DNA to the card matrix, a major laboratory advantage which avoids the freeze-thaw cycles described below.

\subsection{DNA Extraction}

The following reagents were obtained from Qiagen (catalogue numbers in parentheses). For FFPE tissue, five or more $15 \mu \mathrm{M}$ thick sections were placed in a sterile microfuge tube and $300-400 \mu \mathrm{L}$ cell lysis solution (\#158906, $10 \mathrm{mM}$ TRIS-buffer, $\mathrm{pH} 7.5,1 \mathrm{mM}$ EDTA, anionic detergent) plus $2 \mu \mathrm{L}$ of proteinase $\mathrm{K}$ solution $(\# 158918,20 \mathrm{mg} / \mathrm{ml}$ ) were added. Samples were then incubated $4 \mathrm{~h}$ on a shaking thermoblock at $65^{\circ} \mathrm{C}$, which melted the paraffin. Subsequent centrifugation for $5 \mathrm{~min}$ at 13,300 rpm on a table centrifuge separated the resolidified paraffin from the liquid phase, which was then pipetted over to a fresh microfuge tube. Mycobacterial cell walls were then ruptured by three freeze-thaw cycles where for each cycle the microfuge tube was frozen for $5 \mathrm{~min}$ in isopentane on dry ice followed by heating to $80^{\circ} \mathrm{C}$ on a thermoblock for $10 \mathrm{~min}$. Afterwards 1.5 $\mu \mathrm{L}$ of the above proteinase $\mathrm{K}$ buffer solution was added and the tube incubated overnight at $55^{\circ} \mathrm{C}$ on a shaking thermoblock. The temperature was then lowered to $37^{\circ} \mathrm{C}$ and RNA removed by adding $1.5-2.0 \mu \mathrm{L}$ of RNase A solution (\#158922, $4 \mathrm{mg} / \mathrm{ml}$ ) and shaking on a thermoblock for $15 \mathrm{~min}$. Samples were then placed on ice for $1 \mathrm{~min}$ and $100 \mu \mathrm{L}$ of a protein precipitation solution (\#158910: sodium dodecyl sulphate, ammonium chloride and ammonium acetate) was added, the tubes strongly vortexed for $20 \mathrm{sec}$, incubated on ice for $15 \mathrm{~min}$ and centrifuged for $5 \mathrm{~min}$.

DNA remaining in the supernatant was transferred to a fresh tube to which $400 \mu \mathrm{L}$ of pure isopropanol was added and the tube shaken manually, centrifuged for $5 \mathrm{~min}$ (after which a whitish pellet could occasionally be observed) and the supernatant exchanged for $200 \mu \mathrm{L}$ of $70 \%$ ethanol. The mixture was agitated manually, then centrifuged, after which the supernatant was poured out on a Kleenex and the DNA remaining in the tube allowed to dry at room temperature. Rehydration was achieved in $30 \mu \mathrm{L}$ of added EB-DNA hydration solution (\#158914, $10 \mathrm{mM}$ TRIS-buffer $\mathrm{pH} 7.5,1 \mathrm{mM}$ EDTA) at $50^{\circ} \mathrm{C}$ with shaking for at least 10 min. DNA concentration was measured on a microspectrophotometer $\left(\right.$ Nanodrop $\left.^{\oplus}\right)$. 


\subsection{Amplification of IS6110 and 16S-23S rRNA Internal Transcribed Spacer}

MPCR for Mycobacterium tuberculosis (MTC) targeted the IS6110 insert present in the vast majority of isolates in up to 25 copies [5] but lacking in less than $1 \%$ of isolates [6]. We developed two primer pairs labeled with the same 5 -dye (Table 1); these targeted the insert at its 3 ' and 5 ' ends: each primer pair was tested separately, then together; the latter electropherograms yielded congruent peaks of precisely the same size, suggesting that a multiplexed single-tube (Tube 1) mixture could produce an additive IS6110 peak for increased sensitivity.

We chose 14 NTM species/complexes whose 16S-23S intergenic transcribed spacer (ITS) [7] [8] was targeted in Tube 2, which contained a mixture of nine primers. All primer sequences in Tubes 1 and 2 and their dye labels are given in Table 1.

In Table 2, single species and complexes are presented in the first column, followed by the GenBank accession used to determine primer and product sequences in digital multiplexing (VectorNTI Advance ver.10.3), primer combinations annealing for each species template, molecular vs. electrophoretic product size and the corresponding peak color in the last column.

Note that NTM primers (Table 1) have a high degree of sequence homology. Given that NTM taxonomy is unsettled and more than 150 NTM species have been genotyped to date, products derived from primer combinations listed in Table 2 should not be considered definitive species markers for epidemiological study but instead offer a presumptive speciation for clinical guidance. Details of the MPCR are shown in Figure 1.

Table 1. Primer mix: labels and quantities.

\begin{tabular}{|c|c|c|c|c|c|}
\hline \multirow{9}{*}{$\begin{array}{l}\text { Forward } \\
\text { primers }\end{array}$} & Tube no. & $5^{\prime}$ dye & Name & Sequence & $\mu L^{* s}$ \\
\hline & \multirow{2}{*}{1} & \multirow{2}{*}{ FAM } & IS6110 f1 & ACGCTAATTACCCGGTTCAT & 15 \\
\hline & & & IS6110 f2 & AATGCACTAGCCGAGACGAT & 15 \\
\hline & 2 & HEX & MAC f & CACACTATTGGGCCCTGAGACA & 10 \\
\hline & 2 & HEX & MFG f & CACACTATTGGGCTTTGAGA & 10 \\
\hline & 2 & NED & MKG f & CACACTRTTGGGTCCTGAGGCA $^{\dagger}$ & 15 \\
\hline & 2 & HEX & MUCOG f & CACACTGTTGGGTCCTGAGA & 10 \\
\hline & 2 & FAM & SCRO $\mathrm{f}$ & CACACTATTGGGCCCTGAGGC & 10 \\
\hline & 1,2 & FAM & $\beta$-globin $\mathrm{f}$ & CATAAAAGTCAGGGCAGAGCCA & 5 \\
\hline \multirow{6}{*}{$\begin{array}{l}\text { Reverse } \\
\text { primers }\end{array}$} & \multirow{2}{*}{1} & \multirow{2}{*}{-} & IS6110 r1 & CCCGGTTGATGTGGTCGTAG & 15 \\
\hline & & & IS6110 r2 & CGCCGCAGTACTGGTAGAGG & 15 \\
\hline & 2 & - & MAC r & ATGCTCGCAACCACTATCCAAT & 10 \\
\hline & 2 & - & MCAG r & TGCTCGCAACCACTATCCA & 10 \\
\hline & 2 & - & $\mathrm{ABSC} r$ & TGCTCGCAACCACTATTCAG & 10 \\
\hline & 1,2 & - & $\beta$-globin $\mathrm{r}$ & ACTTCATCCACGTTCACCTTGC & 5 \\
\hline
\end{tabular}

${ }^{*}$ From an initial concentration of $100 \mu \mathrm{M}$ for each primer; ${ }^{\varsigma} 40 \mu \mathrm{L} \mathrm{H} \mathrm{H}_{2} \mathrm{O}$ added to IS6110 primer mix; ${ }^{\dagger}$ note single ambiguous nucleotide R. 
Table 2. Target organisms, primers and product size.

\begin{tabular}{|c|c|c|c|c|c|c|}
\hline Species & $\begin{array}{l}\text { GenBank ITS } \\
\text { accession no. }\end{array}$ & $\begin{array}{l}\text { Forward } \\
\text { primer }\end{array}$ & $\begin{array}{l}\text { Reverse } \\
\text { primer }\end{array}$ & $\begin{array}{c}\text { Molecular } \\
\text { target size (bp) }\end{array}$ & $\begin{array}{l}\text { Electrophoretic } \\
\text { size }( \pm \sim 0.5 \mathrm{bp})\end{array}$ & Color \\
\hline M. tuberculosis complex ${ }^{\$ \$ 1}$ & AJ315568 & $\begin{array}{l}\text { IS6110 f1 } \\
\text { IS6110 f2 }\end{array}$ & $\begin{array}{l}\text { IS6110 r1 } \\
\text { IS6110 r2 }\end{array}$ & 148 & 145 & Blue \\
\hline M. avium complex ${ }^{\varsigma 5} / M$. malmoens $e^{\varsigma \varsigma 2}$ & AY701785 & MAC $f$ & MAC r & 103 & 103 & Green \\
\hline M. scrofulaceum ${ }^{\S s}$ & АВ026702 & SCRO $\mathrm{f}$ & MAC r & 104 & 105 & Blue \\
\hline M. fortuitum ${ }^{\S}$ & AF144326 & MFG f & MCAG r & 134 & 137.5 & Green \\
\hline M. kansasii ${ }^{\varsigma \varsigma}$ & DQ134002 & MKG f & MAC r & 106 & 104 & \\
\hline M. ulcerans $s^{\$ \varsigma} / M$. marinum ${ }^{\varsigma s}$ & $\begin{array}{l}\text { AB548734 } \\
\text { AB026701 }\end{array}$ & MKG f & MAC r & 106 & 106 & Black \\
\hline M. chelonae ${ }^{\S}$ & AF144327 & MKG f & MCAG r & 91 & 90 & Black \\
\hline M. haemophilum ${ }^{\S \varsigma} / M$. terra $^{\varsigma \varsigma 3}$ & DQ851570 & MKG f & MAC r & $120 / 121$ & $120 / 121$ & Black \\
\hline M. abscessus ${ }^{\S}$ & AJ314870 & MKG $\mathrm{f}$ & $\mathrm{ABSC} r$ & 91 & 92 & Black \\
\hline M. gordonae $e^{\S s}$ & FJ858761 & MKG f & MAC r & 96 & 96 & Black \\
\hline M. xenopis ${ }^{\text {s }}$ & L15624 & MKG f & MCAG r & 95 & 94 - 96 (4 peaks) & Black \\
\hline M. mucogenicum ${ }^{\varsigma}$ & AY504951 & MUCOG f & MCAG r & 151 & $154+158$ & Green \\
\hline$\beta$-globin. control & NG000007 & $\beta$-globin $\mathrm{f}$ & $\beta$-globin $\mathrm{r}$ & 153 & 151 & Blue \\
\hline
\end{tabular}

${ }^{\mathbf{1}}$ MTC may occasionally be amplified in Tube 2 by MKG $\mathrm{f}$ and MCAG $\mathrm{r}$ to yield a small black peak at $102 \mathrm{bp} .{ }^{2} \mathrm{M}$. malmoense, common in Scandinavia, migrates electrophoretically as MAC. ${ }^{3} M$. haemophilum and M. terrae yield PCR products differing in size by $1 \mathrm{bp}$, which may make distinction equivocal

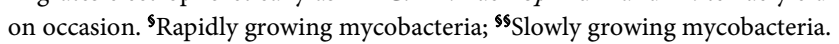

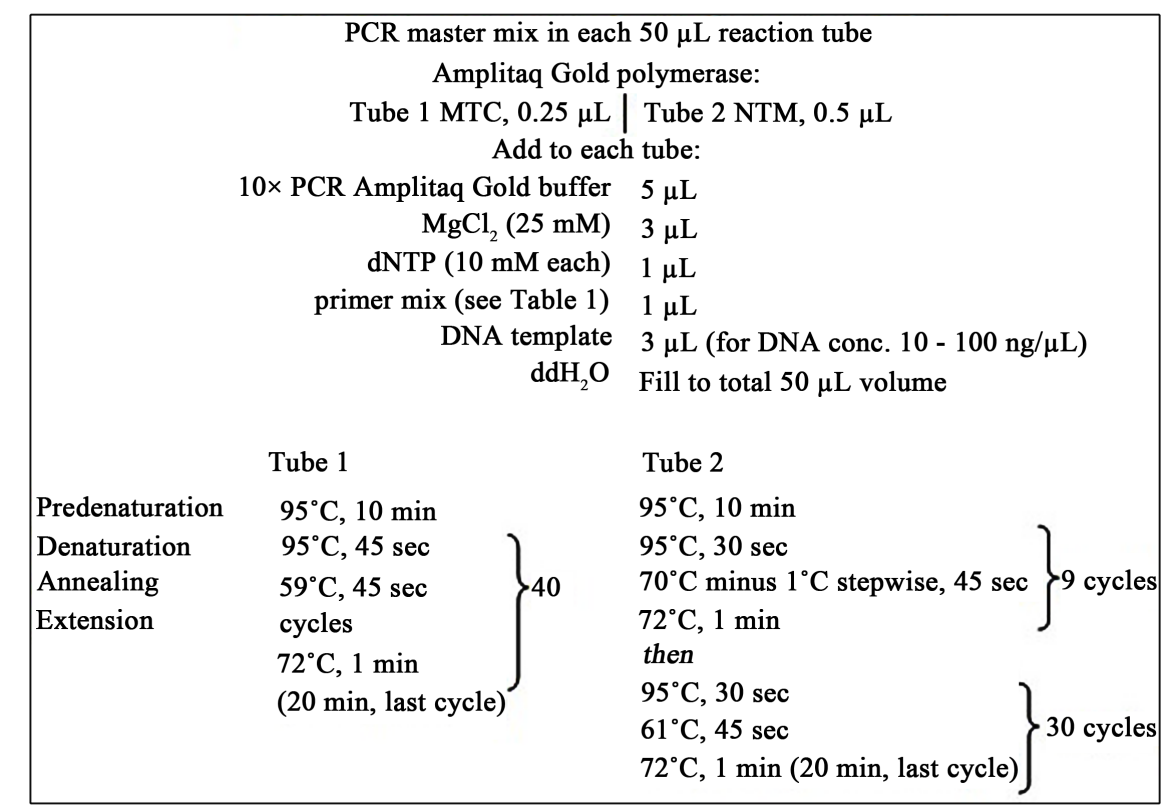

Figure 1. Constituents and parameters for each reaction tube.

\subsection{Capillary Gel Electrophoresis}

One $\mu \mathrm{L}$ of the products was mixed with $12 \mu \mathrm{L}$ formamide and $0.5 \mu \mathrm{L}$ ROX 500 size standards, denatured at $95^{\circ} \mathrm{C}$ for 3 min and run on an ABI Prism ${ }^{\otimes} 3130 \mathrm{Ge}$ - 
netic Analyzer using POP 7 polymer. Loading adjacent capillaries with products from NTM controls and clinical specimens was avoided.

\subsection{Interpreting Electropherograms}

Fragment sizes were determined to within \pm 0.5 bp with GeneMarker ${ }^{\circledast}$ ver. 2 software with peaks rendered in default color mode. Most specimens amplified in Tube 2 lacked a specific NTM peak but showed a single $\beta$-globin control peak; absence of both peaks indicated a nonamplifiable template.

We identified a mycobacterial species if its peak size was within $0.5 \mathrm{bp}$ of the predetermined control size (sixth column in Table 2) and had an amplitude of at least 100 arbitrary fluorescence units (the vast majority of positives surpassed 1000 units). NTM rarely showed double peaks (the signature peak plus an undefined peak not due to excess product) separated by 1 or more bp, a phenomenon described by Gray et al. [8], who amplified the entire 16S-23S ITS of several NTM and presumed extra peaks to represent multiple genomic copies.

\section{Results and Analysis}

Five AFS-positive controls from FFPE tuberculous autopsy tissue yielded a strong MTC peak. Of the 594 diverse specimens, 74 produced a signature MTC peak of the expected size with amplitude $\geq 500$ fluorescence units; included were 17 cytologic specimens submitted on FTA cards, three samples in Hank's solution and one in Preservcyt ${ }^{\mathrm{TM}}$. The remaining 53 positive DNA templates were extracted from FFPE sections.

Infections with common NTM species (such as shown in Table 2) have been tabulated for various tissue sites and the results published [2] with an emphasis on the importance of a diagnosis at the species level which takes into account the clinical setting, radiological findings and especially relevant to this study, histopathological findings consistent with granulomatous inflammation [3]. In the subset of 337 samples also analyzed for NTM (which included 35 confirmed tuberculous samples), 51 were positive; results are given in Table 3. MAC/M. malmoense were the most frequently detected species, whereas lung and skin/soft tissue were the most frequent sites of NTM infection and had the broadest species involvement. These results accord with the above published compilation [2]. Note that M. gordonae, a common contaminant, was detected in specimens from three different sites, including a colon lacking any histological signs of granulomatous inflammation. No peaks consistent with $M$. scrofulaceum, M. mucogenicum or $M$. xenopi were present in our clinical samples.

Double infection with two NTM (MAC and $M$. haemophilum) in skin macrophage infiltrates is illustrated in the electropherogram in Figure 2. Moreover, six cases ( 3 males, 3 females: age range 3 - 94 yrs.) represented double infections with MTC. These NTM included MAC/M. malmoense (3 cases), $M$. kansasii (1), M. marinum/M. ulcerans (1) and M. fortuitum (1). On the basis of these findings, the frequency of simultaneous NTM infection in tuberculous patients was about $17 \%(6 / 35)$. 


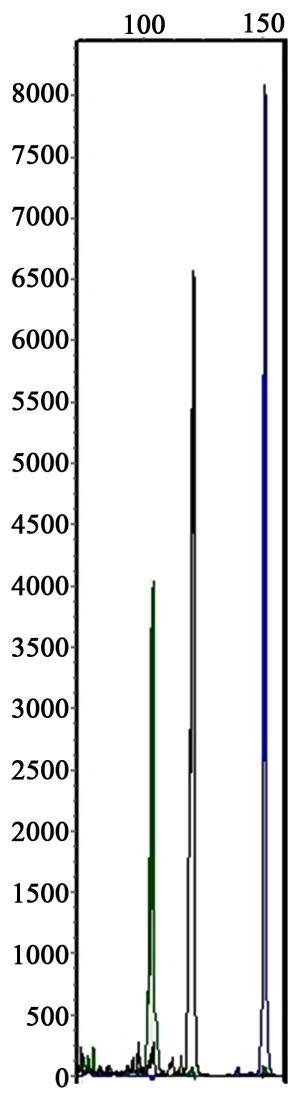

(a)

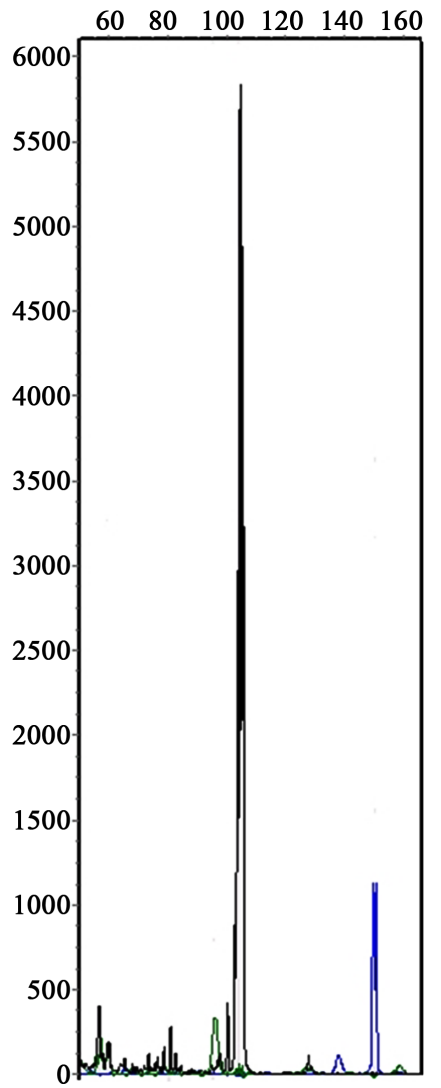

(b)

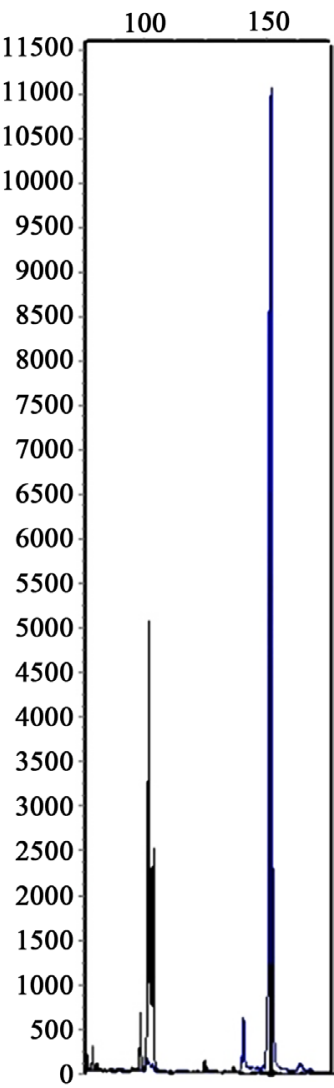

(c)

Figure 2. Representative NTM peaks (see Table 2). Top horizontal axes show product size in bp, vertical axes display arbitrary fluorescence units. Only peaks above 500 units are relevant. Blue corresponds to $\beta$-globin control. (a) Double infection with MAC indicated by the green peak and $M$. haemophilum by the black; (b) Black peak indicates $M$. kansasii; (c) Black migrates as M. marinum or M. ulcerans.

Table 3. Site distribution of NTM $(\mathrm{N}=51)$.

\begin{tabular}{|c|c|c|c|c|c|c|}
\hline Species & Lung & $\begin{array}{c}\text { Lymph } \\
\text { node }\end{array}$ & $\begin{array}{c}\text { Skin/soft } \\
\text { tissue }\end{array}$ & Gastrointestinal & Other & Totals \\
\hline MAC/M. malmoense & 12 & 7 & 3 & 1 & & 23 \\
\hline M. haemophilum ${ }^{\star}$ M. terrae & 2 & & 3 & 1 & 1 & 7 \\
\hline M. kansasii & 2 & 2 & 2 & & & 6 \\
\hline M. fortuitum & & 1 & 1 & & 2 & 4 \\
\hline M. marinum/M. ulcerans & & & 4 & & & 4 \\
\hline${ }^{\star} M$. gordonae & & 1 & 1 & 1 & & 3 \\
\hline M. chelonae & & & 3 & & & 3 \\
\hline M. abscessus & & & & 1 & & 1 \\
\hline Totals & 16 & 11 & 17 & 4 & 3 & 51 \\
\hline
\end{tabular}

${ }^{\star}$ Common contaminant. 
145 paraffin specimens had been stained with $\mathrm{ZN}$ and 9 were positive. Of these, 3 corresponded to MTC, 3 to NTM, while 3 failed to amplify in the MTC tube but were not tested for NTM. Among all ZN-stained paraffin specimens, 36 were positive by MPCR for MTC and/or NTM. Adjusting for 29 MTC-negative specimens which were not studied for NTM, we estimate that our MPCR offers a level of sensitivity about five times that of a standard $\mathrm{ZN}$ stain on formalin-fixed paraffin sections in our laboratory.

Fresh biopsy material from several cases of suspected tuberculosis was submitted elsewhere for PCR or culture. Results discordant with those on our fixed material occurred in five instances: one MTC positive culture from a node in which deparaffinized material was negative in our two-tube PCR, three negatives ( 2 nodes, 1 vertebra) in which unfixed tissue was MTC-positive by another PCR (Serum Institute, Copenhagen) and one lung tissue positive both in an acid-fast stain and our MTC PCR but PCR negative by another method (Serum Institute). We suspect that prolonged formalin fixation times may have contributed to some of the discordance in our paraffin material. Decalcification of bone specimens in organic acids rather than in EDTA severely degrades nucleic acids. This may have led to the false negative MTC MPCR in a case of necrotizing granulomas in a vertebra.

The age distribution of 93 patients (in whom the age was recorded) with our MPCR-confirmed mycobacterial infection is shown in Figure 3. We estimate that half of the MTC cases involved first or second generation migrants from the Middle East, North Africa and to a lesser degree Asia, whereas only one-tenth of NTM cases involved migrants, of whom two were infected with both MTC and

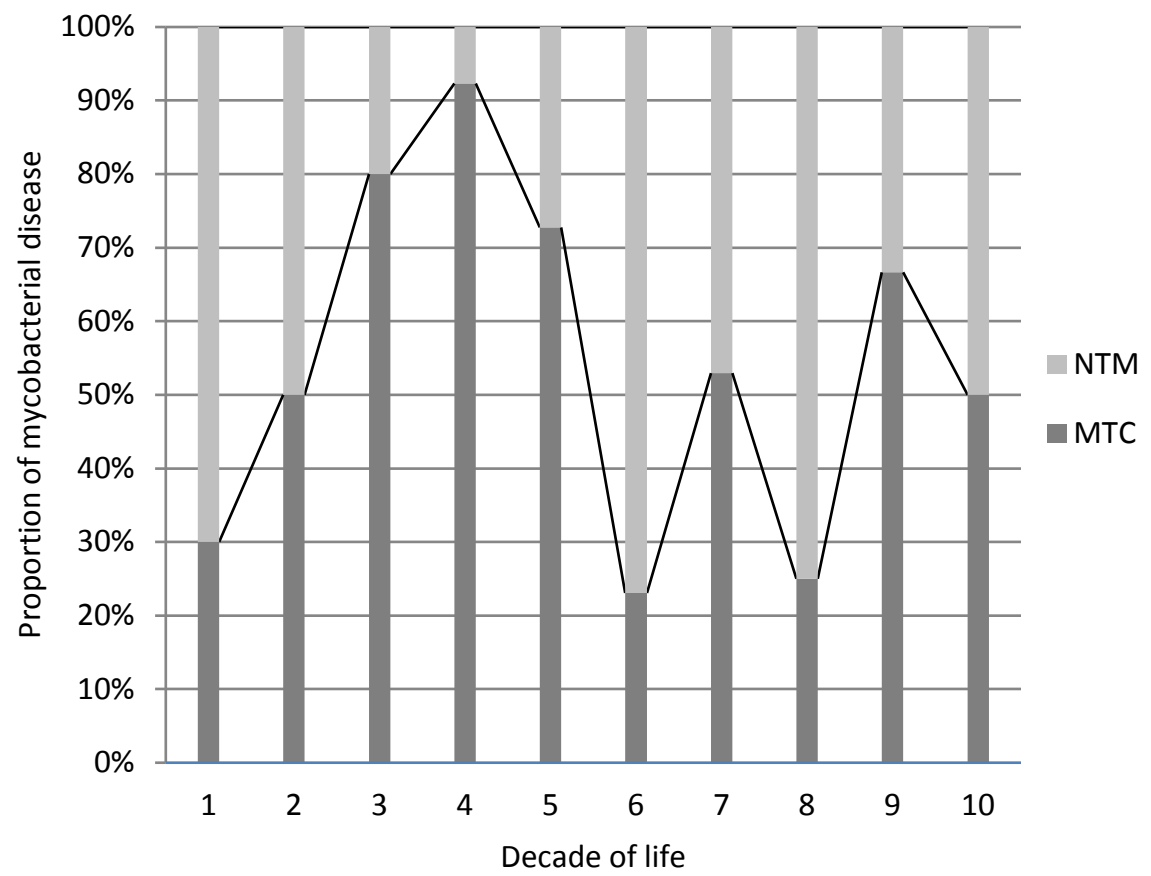

Figure 3. Tuberculous (MTC) vs. nontuberculous mycobacterial (NTM) infection by decade of life. 
NTM. When we queried the departmental database for all 73 instances of identifiable granulomatous reaction in lymph node or lung which were PCR-negative for both TBC and NTM, the estimated fraction of migrant cases from the above regions fell to less than $15 \%$. Thus granulomatous disease in the above populations should be analyzed by sensitive techniques to determine species, most importantly, MTC.

As a proportion of all mycobacterial disease, MTC displayed a roughly bimodal distribution, peaking in the fourth decade of life with a relatively lesser rise in the ninth decade, while NTM infection peaked in the first decade followed by a proportional decline until a resurgence beginning about the sixth decade. However, note that the actual numbers are small and not statistically significant for decennial comparison.

\section{Discussion}

DNA amplification has been used for more than two decades to diagnose mycobacterial infection in cultured specimens as well as fixed paraffinized biopsy tissue. Submitting specimens for mycobacterial culture usually presupposes the possibility of infection, whereas tissue sent for histopathology is often suspected of harboring tumor or other pathology and culturing specifically for mycobacteria may be missed. Cultures require up to three weeks for slow-growing NTM and possibly a further 1 - 2 days to determine species. Turnaround time from tissue submission to sections is about $1-3$ days and perhaps a day more for an acid-fast stain, which has poor sensitivity and offers no indication of species. As experience has accrued, MPCR has become prioritized and the extended 2-tube MPCR is ordered promptly on suspicion of mycobacterial infection. Turnaround is optimally 1 - 2 days.

A second advantage is the ability to amplify cytologic material deposited on an FTA card, which avoids template degradation and extraction procedures. Punches are ready for MPCR after rapid passage through two buffer solutions, thus offering reduction in turnaround time. 123 FTA cards with mainly nodal imprints were submitted, of which 17 (14\%) were positive for MTC. Of 38 cards also tested for NTM, 3 (8\%) were positive. We speculate that adequate template may not always be sampled in punches but is more likely included in paraffin sections. Thus, in a given patient, MPCR may need to be repeated on tissue cuts, if available, and the index of suspicion for mycobacterial infection is high.

The predilection of certain NTM species for specific tissues is well-known and the pattern was largely repeated in our findings. MAC was the dominant species detected in lung and lymph node, while MAC and $M$. haemophilum were the second most frequent just behind $M$. ulcerans/M. marinum in the skin and soft tissue category, although the absolute numbers were small (Table 3 ).

Our finding of the simultaneous occurrence of MTC and NTM in 17\% of adequately analyzed specimens accords with those of Hwang et al. [9], who used various direct molecular techniques plus culturing of respiratory specimens. 
Those authors note that such double infections may have clinical significance in some patients. Others have applied a single-tube MPCR to distinguish MTC from MAC in respiratory material [10]. Although our two-tube MPCR was specifically designed to amplify even degraded templates, and routinely processed sputum and bronchial washings were not tested, the system may be readily adapted for use on respiratory specimens.

\section{Acknowledgements}

M. D. wishes to thank Dr. Jonas Bläckberg, Department of Infectious Disease and Dr. Niels Bendsöe, Department of Dermatology, both in Lund, for supporting the clinical utility of this work.

\section{References}

[1] Fukunaga, H., Murakami, T., Gondo, T., Sugi, K. and Ishihara, T. (2002) Sensitivity of Acid-Fast Staining for Mycobacterium tuberculosis in Formalin-Fixed Tissue. American Journal of Respiratory and Critical Care Medicine, 166, 994-997. https://doi.org/10.1164/rccm.2111028

[2] Griffith, D.E., Aksamit, T., Brown-Elliott, B.A., Catanzaro, A., Daley, C., Gordin, F., et al. (2007) An Official ATS/IDSA Statement: Diagnosis, Treatment, and Prevention of Nontuberculous Mycobacterial Diseases. American Journal of Respiratory and Critical Care Medicine, 175, 367-416. https://doi.org/10.1164/rccm.200604-571ST

[3] Jarzembowski, J.A. and Young, M.B. (2008) Nontuberculous Mycobacterial Infections. Archives of Pathology and Laboratory Medicine, 132, 1333-1341.

[4] Dictor, M., Skogvall, I., Warenholt, J. and Rambech, E. (2007) Multiplex Polymerase Chain Reaction on FTA Cards vs. Flow Cytometry for B-Lymphocyte Clonality. Clinical Chemistry and Laboratory Medicine, 45, 339-345. https://doi.org/10.1515/CCLM.2007.049

[5] Huyen, M.N., Tiemersma, E.W., Kremer, K., de Haas, P., Lan, N.T., Buu, T.N., et al. (2013) Characterisation of Mycobacterium tuberculosis Isolates Lacking IS6110 in Viet Nam. International Journal of Tuberculosis and Lung Disease, 17, 1479-1485. https://doi.org/10.5588/ijtld.13.0149

[6] Lok, K.H., Benjamin Jr., W.H, Kimerling, M.E., Pruitt, V., Lathan, M., Razeq, J., et al. (2002) Molecular Differentiation of Mycobacterium tuberculosis Strains without IS6110 Insertions. Emerging Infectious Diseases, 8, 1310-1313. https://doi.org/10.3201/eid0811.020291

[7] Roth, A., Reischl, U., Streubel, A., Naumann, L., Kroppenstedt, R.M., Habicht, M., et al. (2000) Novel Diagnostic Algorithm for Identification of Mycobacteria Using Genus-Specific Amplification of the 16S-23S rRNA Gene Spacer and Restriction Endonucleases. Journal of Clinical Microbiology, 38, 1094-1104.

[8] Gray, T.J., Kong, F., Jelfs, P., Sintchenko, V. and Chen, S.C. (2014) Improved Identification of Rapidly Growing Mycobacteria by a 16S-23S Internal Transcribed Spacer Region PCR and Capillary Gel Electrophoresis. PLoS One, 9, e102290. https://doi.org/10.1371/journal.pone.0102290

[9] Hwang, S.M., Lim, M.S., Hong, Y.J., Kim, T.S., Park, K.U., Song, J., et al. (2013) Simultaneous Detection of Mycobacterium Tuberculosis Complex and Nontuberculous Mycobacteria in Respiratory Specimens. Tuberculosis, 93, 642-646. https://doi.org/10.1016/j.tube.2013.07.007 
[10] Gopinath, K. and Singh, S. (2009) Multiplex PCR Assay for Simultaneous Detection and Differentiation of Mycobacterium tuberculosis, Mycobacterium avium Complexes and Other Mycobacterial Species Directly from Clinical Specimens. Journal of Applied Microbiology, 107, 425-435.

https://doi.org/10.1111/j.1365-2672.2009.04218.x 